\title{
versants
}

\section{William Ritter et Józef Mehoffer : échange épistolaire autour de l'art slave}

\author{
Noémie CARrauX \\ Université de Lausanne
}

\begin{abstract}
William Ritter et Józef Mehoffer ont entretenu une correspondance pendant soixante ans. Celle-ci débute en 1898 , lorsque l'artiste remporte le concours pour la réalisation des vitraux de la cathédrale de Fribourg. Si leurs premiers échanges sont très formels, les deux hommes vont rapidement se lier d'amitié. L'étude de cette correspondance nous révèle l'importance du rôle qu'a joué William Ritter comme transmetteur du monde artistique slave à l'Europe occidentale entre la fin du XIX ${ }^{e}$ siècle et le début du Xx ${ }^{e}$ siècle.
\end{abstract}

Keywords: William Ritter, Józef Mehoffer, art slave, Sztuka

En I895, Józef Mehoffer, âgé de vingt-six ans, gagne le concours lancé pour la réalisation des vitraux de la cathédrale de Fribourg. Cette œuvre majeure de l'artiste polonais va embrasser l'ensemble de sa carrière, puisqu'il terminera le dernier vitrail en 1936. Le premier à s'intéresser au travail de Mehoffer et à en faire l'éloge dans les journaux est le professeur de théologie fribourgeois Joachim Berthier. C'est probablement lui qui contacte William Ritter, alors à Vienne, pour lui parler du talent de Mehoffer. En effet, au début de l'année I898, Ritter écrit à Mehoffer pour lui demander de lui faire parvenir des photographies des cartons réalisés pour les vitraux de la cathédrale, ainsi que des informations sur ses autres œuvres. En juin de cette même année, William Ritter publie dans La Liberté un article intitulé «M. Joseph Mehoffer au salon de Vienne »; à la suite de cette présentation, Berthier écrit à son auteur pour le remercier d'avoir vengé par ses lignes « ceux qui ont lutté pour le [Mehoffer] faire accepter » (ALS, $n^{\circ} 346$ ). Il faut dire que Ritter ne tarit pas d'éloges quant au travail de Mehoffer, et que, malgré un titre laissant penser que l'article concerne les œuvres exposées à Vienne, il s'efforce essentiellement de convaincre le public fribourgeois que le choix de Mehoffer comme artiste pour les vitraux de la cathédrale relevait du bon sens.

Quand l'art d'un artiste a doté une ville d'une œuvre aussi extraordinairement belle et neuve que les vitraux dont peut, désormais, s'enorgueillir Saint-Nicolas de Fribourg, rien de ce qui intéresse cet artiste ne saurait demeurer indifférent à cette ville. D'autant que, dans le cas particulier, les succès de M. Mehoffer, en une capitale telle que Vienne, sont pour donner 
raison une fois de plus à ceux qui, de tout leur pouvoir, ont appuyé le jeune artiste polonais, et pour causer une grande joie à tous les admirateurs qu'a suscités l'œuvre accomplie. Or, l'exposition des artistes autrichiens à Vienne démontre péremptoirement qu'il n'y a pas, à l'heure actuelle en Autriche, un second peintre de portraits et artiste décoratif de la valeur de M. Mehoffer » (Ritter I898:3).

Cet article inaugure une série qui, durant des années, soutient le même postulat : c'est-à-dire que Józef Mehoffer est le plus grand artiste slave vivant. L'attachement de William Ritter à l'œuvre du peintre et décorateur polonais se comprend par différents facteurs qu'il est possible de saisir en lisant le chapitre que l'auteur neuchâtelois lui dédie dans ses Études d'art étranger (Ritter I906 : I23-I8I). Le critique d'art n'adhère pas uniquement au style de l'artiste, mais, comme c'est souvent le cas chez lui, son attachement artistique est indissociable de ses affects. Il souscrit ainsi non seulement aux caractéristiques esthétiques de l'œuvre de Mehoffer mais également à l'entier de sa personnalité. Par ailleurs, si leur relation débute sur un ton très formel empreint de professionnalisme, les deux hommes vont très rapidement enchaîner les rencontres ${ }^{1}$ et nouer une profonde amitié.

Le jeune artiste polonais présente plusieurs traits qui font écho à la personnalité du critique d'art. Tout d'abord, sa nationalité polonaise, ou, comme il la qualifiera lui-même, sa racialité, rejoint le goût prononcé de Ritter pour ces pays de l'Est européen dans lesquels il entreprend de nombreux voyages. Tous deux, imprégnés des théories racialistes de la fin du XIX ${ }^{\mathrm{e}}$ siècle, analysent les personnalités et l'art au travers du prisme de la race. Dans une longue lettre que Mehoffer adresse à Ritter au mois de février 1906, il lui explique les caractéristiques qu'il considère propres à l'art slave :

Par analogie de ce qu'on peut dire des hommes, on dit des races. Les Slaves c'est la race des individualistes, c'est leur force et aussi la cause de leur faiblesse. Ils ne savent pas développer une activité collective. L'individu chez eux ne veut pas entrer dans le rang et remplir tout simplement la cellule comme le fait une abeille ou un Français par exemple : il exécute des ordres donnés par la mode, il les accepte dans un degré aussi haut comme les autres nations, il reste sous les influences de toutes les écoles tout acceptées par lui mais il les accommode conformément à sa nature plus large de sentiment. Et c'est la base de leur esthétique. Comme artiste il ne ressemble pas à l'artiste français et diffère totalement de l'Allemand. Chez les Allemands l'esthétique une fois acceptée par le corps combattant des artistes règne d'une manière absolue, s'empare de l'industrie et devient industrielle réciproque-

I Première rencontre en I898 à Cracovie, puis la même année à Lemberg. En I90o, Ritter lui rend visite à Florence. Les lettres font également état de nombreuses autres rencontres pour des vacances en Pologne dans la villa d'été des Mehoffer. 
ment et un mot d'ordre, un étendard hissé est soutenu par des milliers de mains. Les Slaves s'ils doivent remplir un rôle c'est de servir de contrepoids par leur don d'individualisme (ALS, n 346, 20 février 1906).

Cette lecture que Mehoffer fait de l'art selon les pays est également celle que Ritter privilégie dans ses Études d'art étranger. Ce qui émerveille le critique d'art, c'est le caractère slave du travail de Mehoffer. Lorsqu'il observe les vitraux de la cathédrale de Fribourg, il n'y voit pas la représentation de personnages de la chrétienté, mais bien celle de figures polonaises. Parlant du vitrail de Notre-Dame-des-Victoires, il ne décrit pas une Vierge à l'enfant, mais « une très grande dame polonaise [...] [qui] porte sur son bras le plus gauche, le plus archaïque, le plus polonais et demi des Petits Jésus » (Ritter 1906 : 137). Il est vrai que Mehoffer déploie dans son œuvre une véritable volonté de créer une forme d'art national polonais. Cet élément est souligné par son adhésion à la Sztuka² : ce mouvement s'appuie sur des représentations du passé d'une Pologne utopique qu'on imagine alors comme traditionnelle. Le réinvestissement de ces motifs sont observables dans le travail de Mehoffer, par exemple dans le vitrail de Sainte-Barbe, où il place en fond une tour de Cracovie; Ritter décrit les vêtements de la Sainte dans les termes suivants : «Elle est vêtue comme on ne l'a peut-être été jamais, et comme pourtant l'imagination se représente que le furent certaines princesses polonaises » (Ritter $1906:$ 153). Dès lors, les vitraux de la cathédrale sont la preuve que "l'art slave n'a plus besoin des leçons de l'Occident " (Ritter 1906 : I62), et que Mehoffer en est le plus prestigieux représentant. D'autres aspects de la production de Mehoffer répondent aux aspirations profondes de l'auteur suisse. Dans ses Études d'art étranger, Ritter relève « ce joyeux choc amical d'un glaive polonais sur le sobre écu noir et blanc d'une si patricienne simplicité de la République fribourgeoise, [qui l'] enchante comme le cliquetis d'art le plus symbolique et pour [lui] le plus impressionnant qui [le] puisse émouvoir, puisqu'il émeut en [lui] tout ce dont [il est] fait : le passé des vieilles villes allemandes et l'impérieux rêve, l'impériale volonté de [se] slaviser» (Ritter 1906: I36-I37). Cette citation nous donne plusieurs clés pour comprendre l'attachement des deux hommes. L'art de Mehoffer n'est pas uniquement un art polonais, mais il s'inscrit dans le mouvement du symbolisme, le courant artistique favori de Ritter, qui n'hésite pas à comparer le travail du Polonais à celui de Puvis de Chavannes, Segantini, Brangwyn, Boecklin et Stucks (Ritter I898 : 3). En outre, l'aveu de son «impériale volonté de [se] slaviser » révèle le goût de Ritter pour les pays slaves, mais également pour la noblesse. Or, Mehoffer est issu d'une famille aristocratique, et il vit dans le contexte monarchique de l'Empire aus-

2 La Sztuka est le pendant polonais de la Sécession viennoise. Elle est créée en I897 et a pour but d'introduire l'art polonais sur le plan international. 
tro-hongrois. Enfin, en plus d'être slave et noble, Mehoffer est catholique, ce qui entre en résonance avec l'éducation et la culture de Ritter, formé en France chez les Jésuites. Ces différents éléments vont conduire Ritter à adhérer complètement au travail de Mehoffer.

La relation des deux hommes comprend trois étapes. Tout d'abord, la phase de découverte par Ritter de l'art de Mehoffer va le pousser à consacrer de nombreux articles louangeurs à l'artiste polonais. Durant cette première période, Mehoffer profite de la position et des relations de Ritter en Europe pour se créer un réseau, les articles du critique permettant à l'artiste de se faire connaitre en dehors des frontières austro-hongroises. Dans ses lettres, il se montre extrêmement reconnaissant envers Ritter. Lorsqu'il obtient la médaille d'or à l'Exposition de Paris en I9oo pour son portrait La Cantatrice, il n'oublie pas de rappeler à Ritter qu'il a été « le premier à prendre le patronage de ce portrait " (ALS, ${ }^{\circ}$ 346, 25 juin 1900). Dans la même lettre, Mehoffer lui demande des conseils pour son voyage parisien, et s'il est possible de l'introduire dans les cercles influents de la capitale française. En 1900 encore, Mehoffer est nommé à la chaire de dessin de l'Académie des beauxarts de Cracovie : grâce à l'appui de William Ritter, son travail est désormais reconnu tant à l'étranger que chez lui. Les années suivantes vont faire entrer les deux hommes dans la deuxième phase de leur relation. Si jusque-là Mehoffer a pu profiter des articles et du réseau de Ritter pour se forger une certaine notoriété, un mouvement inverse semble s'amorcer, une fois la popularité de l'artiste établie. En effet, en mai 1906, c'est au tour de Mehoffer de répondre à une sollicitation de Ritter, qui cherche à s'introduire dans le cercle des peintres russes. L'artiste lui donne des conseils, jouant vis-à-vis de Ritter le rôle que ce dernier avait joué pour lui ; il lui parle également des différentes expositions qui se déroulent dans les pays slaves, et des artistes auxquels il faudrait s'intéresser. Obéissant à un double mouvement, cette relation s'apparente à une sorte de pivot pour les échanges artistiques entre l'Europe occidentale et les pays slaves. La publication des articles de William Ritter sur les productions artistiques slaves dans des journaux d'Europe occidentale semblent jouer un rôle fondamental dans la diffusion de ces modèles au-delà de leur propre frontière et dans leur acceptation à l'étranger comme dans les pays d'origine, ainsi que l'explique Mehoffer dans sa lettre du 20 février 1906 :

La production artistique slave [...] doit chaque fois subir l'examen à l'étranger ; si elle n'est pas bien comprise et appréciée là-bas, tant pis pour elle, elle sera mal reçue dans son propre pays qui a la faiblesse de ne pas juger par lui-même et qui se décide difficilement à l'enthousiasme pour ses œuvres d'art. Or, cher Monsieur, voyez que votre activité dans ce champ de bataille slave est bien accueillie par nos artistes et vos sorties dirigées à l'adresse de l'art allemand là où il présente ses côtés faibles nous sont très utiles (ALS, n 346, 20 février 1906). 
L'importance que la critique d'art de Ritter revêt pour les milieux de l'art slave apparaît clairement au moment où la relation entre les deux amis entre dans sa troisième phase. En 1908, Mehoffer convie le critique d'art à l'exposition de la Sztuka à Vienne. Cette exposition présente 358 œuvres de ses membres dont un hommage posthume rendu à Wypianski. Après sa visite, Ritter écrit plusieurs articles dans des revues d'art en France, en Italie, en Bohême et en Suisse. Dans ceux-ci il fait, comme à son habitude, l'éloge du travail de Mehoffer, le désignant comme le chef de file du mouvement dont il vante les mérites.

L'Autriche n'a à l'heure actuelle pas de gloire artistique moins contestable que sa jeune école polonaise, née du vieux maître Jan Matejko, ce "grand Vénitien de Cracovie" et de l'excellent paysagiste Jan Stanislawaski, le peintrepoète émule de Mehoffer, au premier rang de la nouvelle génération. Celui-ci prend désormais la tête de l'école. Je suis en mesure d'affirmer, et je le fais de toutes mes forces, que c'est le plus grand artiste aujourd'hui vivant sur toute l'étendue de la monarchie austro-hongroise. Ni Klimt à Vienne, ni Jan Pressier à Prague, qui seuls pourraient lui être opposés, n'atteignent à la splendeur abondante et aisée de son goût décoratif, à la santé matérielle de ses travaux de peintre, non plus qu'à la variété cossue, à la hardiesse enjouée, à la facilité débordante de son œuvre déjà innombrable. Disons-le sans hésiter, c'est le génie dans toute la force du terme, le génie rayonnant, absolu, aisé et souriant, qui n'a rien à chercher pour trouver toujours la splendeur nouvelle, pour concevoir sans cesse ce à quoi personne n'avait encore pensé (Ritter $1908: 2-3$ ).

Comme le préfigure cet extrait, la plus grande partie de l'article est consacré à Mehoffer ; Ritter y laisse volontairement de côté certains artistes présents dans l'exposition, prétendant ne pas avoir suffisamment de place pour s'y attarder. La focalisation sur Mehoffer et le silence volontaire entourant d'autres noms mènent à une cabale contre l'artiste polonais, qui est même provoqué en duel. En effet, ses collègues de la Sztuka l'accusent d'avoir influencé William Ritter dans la rédaction de son article. C'est Stanislas Estreicher qui informe le critique de la situation difficile dans laquelle se trouve son ami :

M. Mehoffer a été dans les derniers temps l'objet d'action hostile de la part de quelques collègues de l'académie des b. arts, qui ont rompu les relations avec lui en l'accusant de vous avoir inspiré dans vos articles sur Sztuka à son profit - et spécialement pour nuire à Pankiewick et M. Wyczolkowski (ALS, $\mathrm{n}^{\circ} 346, \mathrm{I} 4$ juin $\left.\mathrm{I908}\right)$. 
L'affaire finit par se tasser, mais Mehoffer et sa femme ${ }^{3}$ restent profondément marqués par cette déconvenue, décidant dès ce moment de "ne fréquenter plus personne pour avoir la paix » (ALS, $n^{\circ} 346$ ). Les échanges entre les deux hommes iront en diminuant. L'entrée en guerre de l'Europe joue évidemment un rôle dans ce déclin. L'art de Mehoffer connaît, quant à lui, une certaine forme de stagnation, qui s'explique sans doute par ces deux mêmes facteurs - soit le traumatisme personnel provoqué par cette mésaventure, et les retombées de la Première Guerre mondiale.

L'étude de l'échange épistolaire entre Mehoffer et Ritter nous montre une forme de destin lié, puisque le contact entre leurs milieux artistiques respectifs n'aurait pu être possible sans, dans un premier temps, l'intérêt de Ritter pour le travail de Mehoffer, et dans un deuxième temps, sans les conseils du second au premier. Le poids du critique d'art helvétique dans la transmission à l'étranger des œuvres artistiques du monde slave est confirmé par la cabale dont est victime le couple Mehoffer. Les articles de William Ritter font la renommée de Józef Mehoffer à l'étranger et en Pologne, tout en jouant un rôle dans la diffusion européenne des modèles artistiques slaves. Durant ces années, William Ritter est le porte-voix européen du milieu de la Stzuka qui, sans lui, reste dans l'ombre de Sécession viennoise. Si l'auteur suisse permet à l'artiste polonais de se faire connaître, il participe également d'une certaine manière à son déclin. Par-delà le fait que la trop grande attention portée à Mehoffer finit par lui porter préjudice, il semble que Ritter et lui soient véritablement faits du même bois. Leurs similitudes sont telles que ni l'un ni l'autre ne réussit à adapter son style aux attentes de l'époque et du public, lorsque le symbolisme est dépassé. Tous deux inadaptés aux nouveaux codes esthétiques du début du siècle, ils vont désormais incarner une image de dandy en décalage avec leur temps.

\section{Bibliographie}

Berthier, Joachim J., « Les vitraux de Saint-Nicolas », La Liberté, 25 juin I898, p. 3. —. « Beaux-Arts », La Liberté, I3 décembre 1896, p. 2.

—. « Notre Dame de la Victoire (Nouveau vitrail de M. Mehoffer à Saint-Nicolas de Fribourg)", Fribourg artistique, 1898, pp. XIII.

Bourgarel, Gerard [et al.], Józef Mehoffer : de Cracovie à Fribourg, ce flamboyant art nouveau polonais, Fribourg, Méandre, 1995.

Fabelova, Karolina, «La "fusion des arts" et la "recherche de la modernité". L'introduction en Bohême d'un chapitre de la critique d'art française :

3 L'épouse de Mehoffer, également proche de Ritter puisque celui-ci a passé à plusieurs reprises des vacances avec le couple, a également été accusée d'avoir influencé le critique d'art. 
Camille Mauclair et sa relation à F. X. Salda et William Ritter ", Revue des études slaves, tome 74, fascicule I, 2002, pp. 63-76.

Kaenel, Philippe, "William Ritter (I867-I955) : un critique cosmopolite, böcklinien et anti-hodlérien ", Revue suisse d'histoire, $\mathrm{n}^{\circ} 48$, cahier I, I998, pp. 73-98.

Reynold, Gonzague de, «L'art religieux fribourgeois », La Liberté, 22 mars I909, p. I.

Ritter, William, "Chose d'art », Revue générale, août I895, pp. I-I5.

—. « M. Joseph Mehoffer au salon de Vienne », La Liberté, 25 juin I898, p. 3.

-. "Correspondance d'Autriche, les expositions universelles de Vienne ", Gazette des beaux-arts : courrier européen de l'art et de la curiosité, juillet I898, pp. I65-I76.

—. "Lettres tchèques ", Mercure de France : série moderne, I octobre 1905, pp. 462-469.

-. "Correspondance de Munich : la IX exposition internationale des beaux-arts ", Gazette des beaux-arts : courrier européen de l'art et de la curiosité, janvier 1906, pp. 70-82.

—. «Un peintre polonais : M. Josef Mehoffer », Études d'art étranger, Paris, Mercure de France, I906, pp. I23-I8I.

-. "L'exposition de l'art chrétien moderne à Vienne », La Chronique des arts et de la curiosité, supplément de la Gazette des beaux-arts, 3 février 1906, pp. 36-37.

-. «Correspondance de Vienne : les artistes polonais de la société Sztuka au Hagenbund ", Gazette des beaux-arts : courrier européen de l'art et de la curiosité, janvier-juin I908, pp. 433-44I.

—. «L'art polonais à Vienne », L'Art et les Artistes : revue mensuelle d'art ancien et moderne, avril I908, pp. I74-I79.

—. « Joseph Mehoffer à l'exposition polonaise de Vienne », La Liberté, I8 avril I908, pp. 2-3.

Von Roda, Hortensia, Die Glasmalerein von Józef Mehoffer in der Kathedrale St. Niklaus in Freiburg [französiche Übers. (résumé) Catherine Courtiau, Isabelle Fichenberger], Bern, Gesellschaft für Schweizerische Kunstgeschichte, I995.

Zenczak, Anna, « Jeunesse de l'art, un vitrail créé en 1900 par Józef Mehoffer », Revue suisse d'art et d'archéologie, n 5I, cahier I, I994, pp. 23-38. 